\title{
Diurnal variation of corneal sensitivity
}

\author{
MICHEL MILLODOT \\ School of Optometry, University of Montreal, Canada
}

The continuous wear of contact lenses affects corneal sensitivity. A reduction of corneal sensitivity to touch takes place and seems to become greater with the number of hours of wear, becoming significant after 6 to 8 hours of wear. This phenomenon has been demonstrated by several authors and was reviewed by Millodot (I97I). The measurements are carried out before inserting the lens and just after removal. However it is not known how the sensitivity of the cornea would vary throughout the day if the patient were not wearing contact lenses, and the present study was designed to determine the diurnal variation of corneal sensitivity in persons who do not wear contact lenses. Such data provide the control experiment against which the reduction of corneal sensitivity in contact lens wearers must be compared. It will be shown that corneal sensitivity does not diminish throughout the day in the control eyes and that, therefore, the contact lenses must account for the reduction. Moreover, the normal eye actually becomes more sensitive by the evening, so that the reduction brought about by wearing contact lenses is even greater than it appears to be when the sensitivity is measured in the same eye before and after wearing the lens.

\section{Material and method}

The subjects were divided into two groups. The first group consisted of 7 persons ( 6 females, I male) and the second group of 5 persons ( 3 females, 2 males). All had healthy eyes and did not wear contact lenses. Their ages ranged from 19 to 25 years.

The aesthesiometer used was that of Cochet and Bonnet (1960) which is based upon the instrument devised by Boberg-Ans (1955). It consists of a nylon monofilament $0 \cdot 12 \mathrm{~mm}$. in diameter which may be varied in length so that the pressure applied against the cornea may range from I I mg. $200 \mathrm{mg} . / 0 \cdot 0113 \mathrm{~mm}^{2}$. It is directed perpendicularly against the cornea until the first visible bend can be seen. A corneal point near the limbus in the 6 o'clock position was first tested, and this was followed by measurements in the centre of the cornea. During the measurements the patient remained seated, his head resting on a chin rest and his eyes directed at a fixation target near the ceiling when the peripheral corneal point was tested. Because the nylon monofilament is affected by humidity (Millodot and Larson, 1967), all measurements were taken when the humidity in the room was between 30 and 40 per cent. The subjects of the first group were each tested on both eyes at about 9 a.m., 12 a.m., 3 p.m., 6 p.m., and 9 p.m. The subjects of the second group were each tested on both eyes at $9 \mathrm{p} . \mathrm{m}$. and at $9 \mathrm{a} . \mathrm{m}$. the next morning. This second group served as a control for possible habituation to the repeated measurements throughout the day with the first group. Moreover the data cover a period of 24 hours.

The touch threshold of the cornea was determined by the use of the method of constant stimuli. These measurements always begin with the lowest pressure; four or six contacts are made with the cornea as well as at least one blank to test the subject's reliability. A percentage of correct answers is obtained by asking the subject how many times he felt the probe. The operation is repeated 
with usually three more values of the pressure. From these data, the touch threshold (in mg./area) for 50 per cent. correct responses is read on a graph relating the percentage of correct responses to the pressure. The aesthesiometer is scaled in length units, which must be transformed into pressures (in mg./area). Our instrument had been previously calibrated on a very sensitive scale and a calibration curve relating length and pressure was drawn so that units of length as low as $\mathrm{I} \mathrm{mm}$. could be transformed into pressure (Millodot, r969).

\section{Results}

EXPERIMENTAL GROUP

The measurements made at the centre of the cornea gave a median threshold of I I mg./area for the fourteen eyes at 9 a.m. Because of the limitation of the aesthesiometer it was not possible to quantify the central threshold throughout the day since the next sets of measurements provided thresholds which were lower than I I mg./area. Nevertheless the central area of the cornea displayed an increase in sensitivity after 9 a.m. The touch thresholds at the peripheral point of the cornea measured throughout the day are given in the Figure (continuous line), each point representing the mean touch threshold for the fourteen eyes. The results display an increase in sensitivity (threshold $-\mathrm{I}$ ) throughout the day for this sample of subjects, the greatest decline being between 9 a.m. and 12 noon. The difference between the means of the thresholds at 9 a.m. and 9 p.m. is significant $(t=I \cdot 8 ; P<$ $0 \cdot 05$ ); that is, the eye has become more sensitive by the end of the day and is least sensitive in the morning.

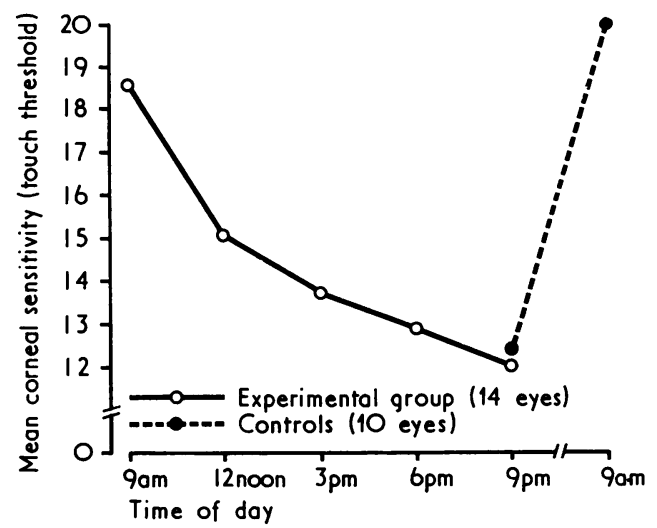

FIGURE Threshold of corneal sensitivity to touch (mg./sq.mm.) as a function of the time of day

GONTROL GROUP

The measurements in this group, beginning in the evening, were not quantifiable in the centre of the cornea since most subjects felt the minimum pressure available. The second set of measurements made the next morning showed a decrease in sensitivity, and the median threshold for these ten eyes was $12 \mathrm{mg}$./area. The results obtained at the peripheral point of the cornea are given in the Figure (broken line). The points represent the mean touch threshold for the ten eyes at 9 o'clock in the evening and 9 o'clock in the morning. A significant difference $(\mathrm{t}=3.28 ; \mathrm{P}<0.005)$ was found between the two means, the greater (that is less sensitive) being in the morning. These subjects had never been tested for this sensation before and the results cannot have been affected by habituation. Hence we can accept the increase in sensitivity found in the experimental group throughout the day. Moreover, the thresholds for the contact group were very close to those obtained 
for the experimental group both in the morning and in the evening. The present results suggest that there exists a diurnal variation of corneal sensitivity for most subjects since the phenomenon was not present in one of the subjects of the experimental group.

\section{Discussion}

The existence of a diurnal variation of corneal sensitivity does not appear to have been illustrated hitherto. It is characterized by a lower sensitivity to touch in the morning, a progressive increase in sensitivity towards the evening, and a return to the lower level by the next morning. This diurnal variation is somewhat analogous to the variation in intraocular pressure, which is greater in the morning and decreases throughout the day. It may be that corneal sensitivity is partially related to the intraocular pressure. The higher pressure within the eye could cause a neural adaptation of the dendrites innervating the cornea, thus increasing the touch threshold (i.e. lower sensitivity). Indeed it is known that a large increase in intraocular pressure as found in glaucoma markedly reduces corneal sensitivity (Boberg-Ans, 1955). The diurnal variation of intraocular pressure is of the order of 4 or $5 \mathrm{~mm} . \mathrm{Hg}$, but it is not known whether this slight rise in pressure would be sufficient to alter the sensitivity of the cornea.

Mandell and Fatt (1965) found that the cornea was oedematous (whether through epithelial oedema or oedema of the whole cornea is not specified) while the eye was closed and became thinner upon awakening. They attributed the thinning to evaporation of water from the tears producing a hypertonic film which withdrew water from the cornea by an osmotic effect. The cornea regained its original thickness within an hour of opening the eyelid, at least for the subject of their experiment. This swelling of the epithelial cells must stimulate the nerve endings and thereby produce some neural adaptation.

The wearing of hard contact lenses gradually results in a reduction of corneal sensitivity, so that after 8 hours' wear the cornea is usually much less sensitive than before the lens: was inserted. In view of the present results, the reduction in corneal sensitivity after wearing contact lenses is even greater than has been hitherto believed, since the normal cornea becomes more sensitive by the end of the day. It is therefore important to warn patients against their wearing contact lenses too long. This suggestion applies principally to hard lenses for almost no reduction of corneal sensitivity was noted in wearers of hydrophilic lenses (Knoll and Williams, 1970; Larke and Sabell, 1971).

Finally, the fact that the cornea appears to be less sensitive in the morning may be of some value to practitioners in planning appointments for these patients. It should, however, be noted that the diurnal variation in corneal sensitivity reported here concerns young adults but may not apply to older persons in whom the corneal sensitivity is greatly reduced.

\section{Summary}

Corneal sensitivity in young adults was measured at different times throughout the day. In one group measurements began in the morning and ended in the evening. In a second group one measurement was made in the evening and another the next morning.

The results showed that corneal sensitivity was lowest in the morning and highest in the evening and that these findings were not due to habituation. An attempt is made to explain this fluctuation which is analogous to and may be related to that of the intraocular pressure. The corneal oedema when the subject is asleep may also help to account for this fluctuation. 
The author thanks Mr. Henry Szikman for his help in carrying out this investigation.

\section{References}

BOBERG-ANS, J. (I955) Brit. 7. Ophthal., 39, 705

COCHET, P., and BonNet, R. (1960) Clin. ophtal., 4, 3

KNoll, H. A., and williams, J. (i970) Amer. 7. Optom., 47, 56 I

LARKe, J. R., and SABEll, A. G. (I97I) Optician, r62, July 23, p. 8

MANDELL, R. B., and FATT, I. (1965) Nature (Lond.), 208, 292

MILlodoT, м. (1969) Optician, 157, 267

( 197 I) Ibid., 162, December 3 I, p. 23

and Larson, w. ( I967) Contact Lens (Lond.), r, 5 